\title{
69. STRUCTURE AND DYNAMICS OF BARRED SPIRAL GALAXIES WITH AN ASYMMETRIC MASS DISTRIBUTION
}

\author{
G. DE VAUCOULEURS \\ University of Texas, Austin, Tex., U.S.A. \\ and \\ K. C. FREEMAN \\ Australian National University, Canberra, Australia
}

\begin{abstract}
The asymmetric structure and dynamics of late-type barred spirals is analyzed in terms of a model consisting of a small prolate spheroid (the bar) displaced from the center of a large oblate spheroid (the disk). The non-axisymmetric motions predicted for this model are in remarkable agreement with optical and radio observations of internal motions in several magellanic barred spirals, including NGC 4027 and the Large Cloud. In particular, the model explains the displacement of the apparent center of the rotation curve from the center of the bar.
\end{abstract}

1. Until recently dynamical models of galaxies rested on the assumption of circular symmetry for ordinary spirals or, at least for barred spirals, of axial symmetry. In general these assumptions appear reasonable for isolated galaxies; for example the luminosity distribution in an ordinary spiral such as M33, type SA(s)cd, is very nearly symmetric about its nucleus at the rotation center (Figure 1a). Nevertheless there is evidence that even in such apparently symmetric systems, a small fraction of the mass, including both stars and gas, is significantly displaced form the center of symmetry. For example in M33 the centroid of the distribution of supergiant stars brighter than $m_{\mathrm{B}}=20.2\left(M_{\mathrm{B}}<-4.5\right)$ (de Vaucouleurs, 1961b) is displaced from the nucleus by some $2^{\prime}$ to $3^{\prime} \simeq 500 \mathrm{pc}$ in position angle $200^{\circ}$ (Figure $1 \mathrm{~b}$ ). Similarly, the center of gravity of the integrated $21 \mathrm{~cm}$ brightness distribution (Gordon, 1969) is displaced from the optical nucleus by about $4^{\prime} \simeq 800$ pc in p.a. $220^{\circ}$ (Figure 1c). This direction is unrelated to that of M31 which is in p.a. $315^{\circ}$. Here the excess mass involved is a very small fraction of the total mass of the galaxy, probably less than $0.1 \%$.

A more pronounced offset exists in M101 where the distribution of both $21 \mathrm{~cm}$ and optical emission is conspicuously asymmetric (Beale and Davies, 1969). Here the mass asymmetry may involve as much as several per cent of the total mass, with $1 \%$ in the gas alone, but in this case it may be more a result of tidal interaction with NGC 5474 and other members of the M101 group rather than a manifestation of basic structural asymmetry.

2. In late-type barred spirals, and in particular of the Magellanic type, i.e. for types SB(s)d to SB(s)m of the revised Hubble system (de Vaucouleurs, 1959b), asymmetry is a basic and characteristic property of the mass distribution clearly visible on direct photographs (Figure 2a) and reflected in the surface distributions of practically all components of the system, in particular supergiant stars, neutral hydrogen, luminosity etc. (Figure 2b). Here the asymmetry involves several per cent of the total mass of the 

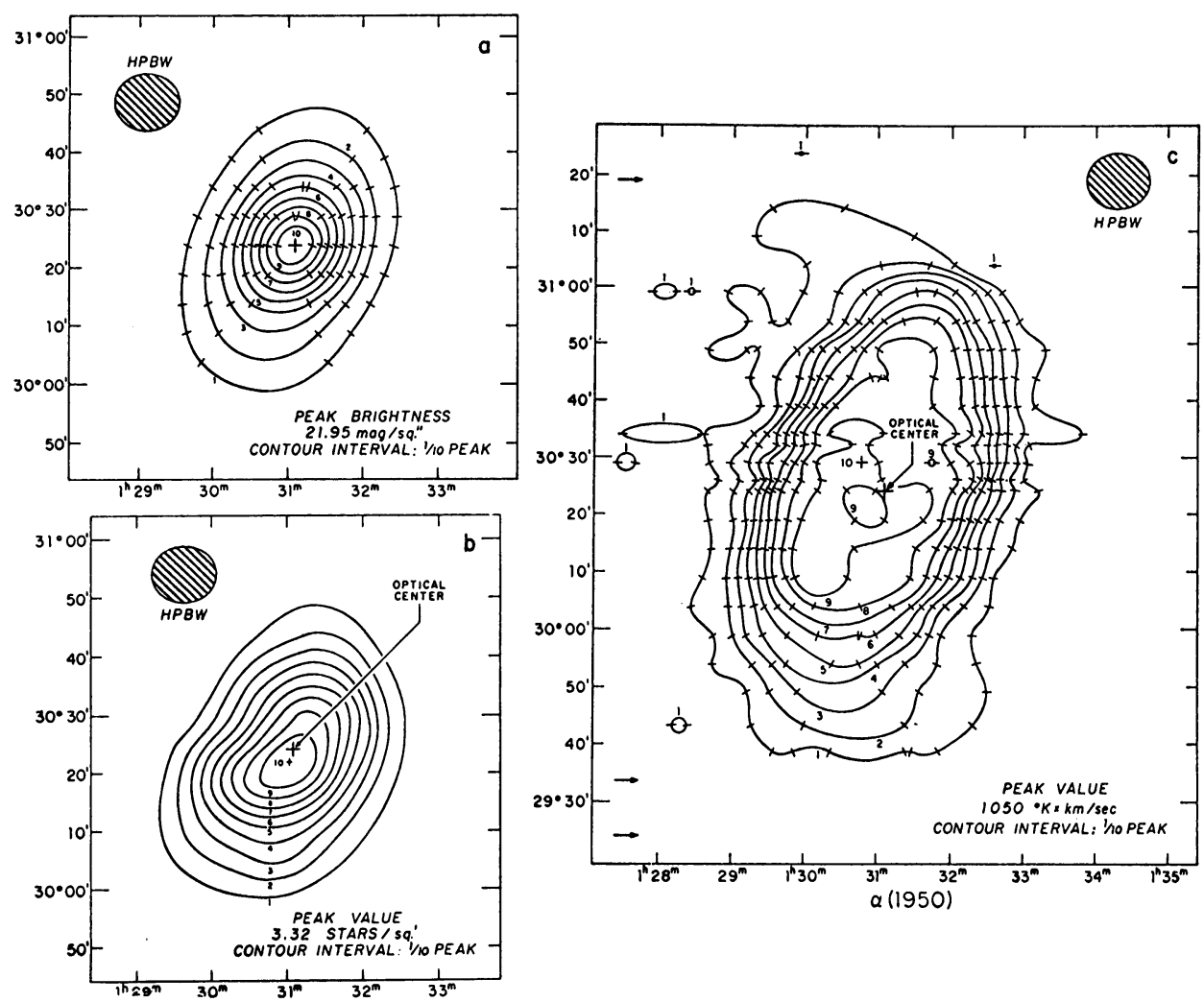

Fig. 1. Comparison of optical and $21 \mathrm{~cm}$ distributions in M33. - (a) Blue light integrated luminosity in B system, after de Vaucouleurs (1959a); - (b) Blue light star counts to $m_{\mathrm{B}}=20.2\left(M_{\mathrm{B}}<-4.5\right)$, after de Vaucouleurs (1961b); - (c) $21 \mathrm{~cm}$ integrated flux density, after Gordon (1969). (a) and (b) were convolved with 300-foot NRAO antenna beam to match resolution in (c). Note excess of supergiant stars (b) and neutral hydrogen gas (c) in quadrant ssf nucleus which coincides with center of optical isophotes (a).

system and gives rise to the puzzling asymmetry of the rotation curve with respect to the center $C$ of the bar first noted in the Large Magellanic Cloud (Kerr and de Vaucouleurs, 1955; Feast, 1964). Later the same effect was observed in several other systems of closely related types, in particular NGC 55 (de Vaucouleurs, 1960; Robinson and Van Damme, 1966), NGC 4027 (de Vaucouleurs et al., 1968), and NGC 4631 (G. and A. de Vaucouleurs 1963; Roberts, 1968; Crillon and Monnet, 1969). In all these systems the displacement from $C$ of the center of symmetry $C_{\mathrm{r}}$ of the rotation curve is on the order of 0.5 to $1.5 \mathrm{kpc}$ toward the richer side of the system. This is also the side marked by the major spiral arm $B_{1}$ giving to some systems the superficial appearance of 'one-armed' spirals, although closer inspection always reveals the presence of one or more additional arms. The basic structure of these asymmetric barred spirals is shown in Figure 2a. The so-called 'radio center' $C_{\mathrm{r}}$ of the rotation curve was originally introduced by Kerr and de Vaucouleurs 
o.
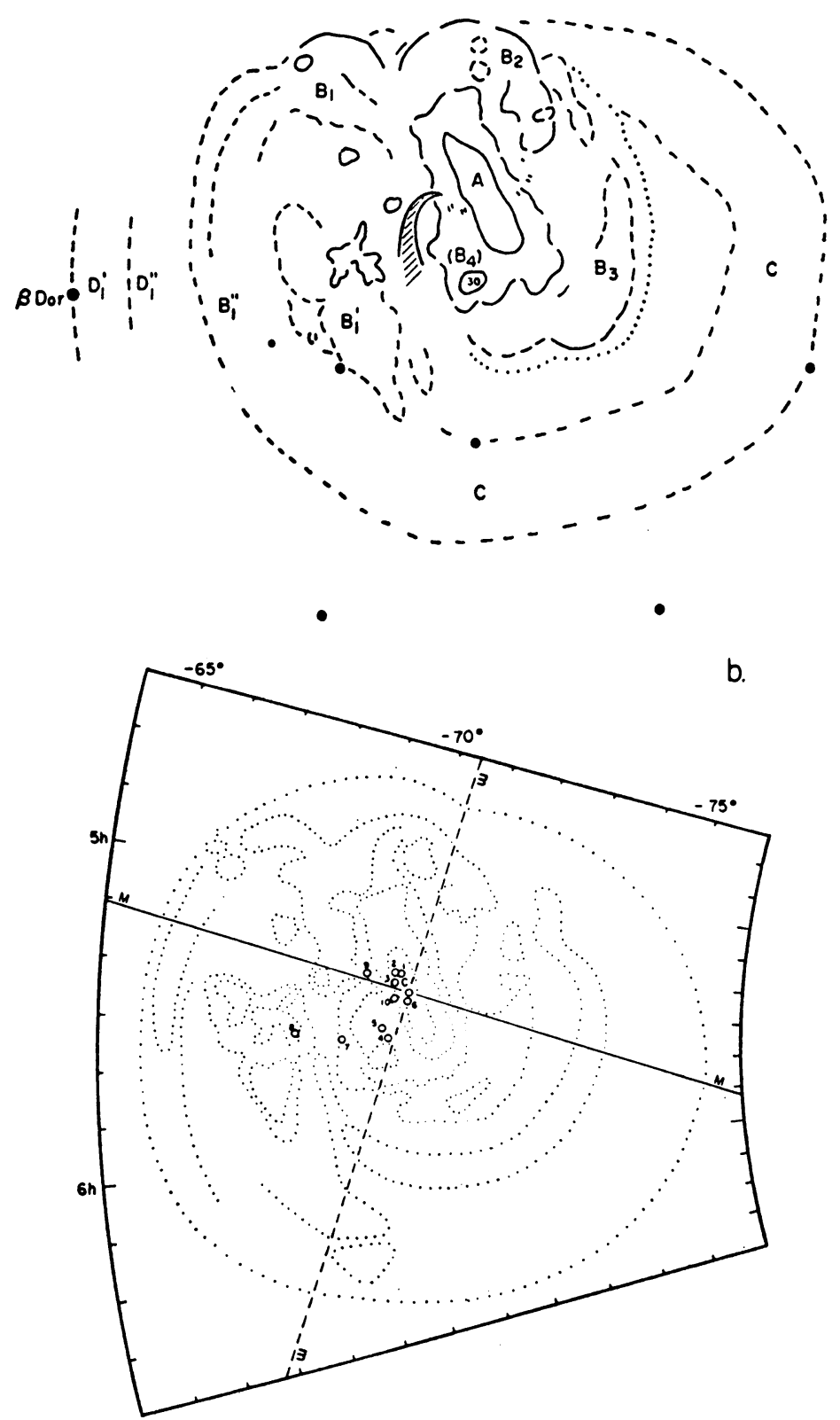

Fig. 2. Asymmetric structure of magellanic-type barred spirals. -(a) Key to barred spiral structure of Large Magellanic Cloud showing axial bar $A$, inner spiral arms $B_{1}$ to $B_{3}$, outer loop $C$, and outlying spiral $\operatorname{arcs} D_{1}^{\prime}{ }_{1} D^{\prime \prime}{ }_{1}$. - (b) Locations of various distribution centroids in Large Magellanic Cloud. $C=$ center of symmetry of bar. $1=$ surface brightness peak in blue light, $2=$ centroid yellow light isophotes, $3=$ centroid of planetary nebulae, $4=$ geometric center of outer loop, $5=$ centroid of outlying clusters, $6=$ centroid of all clusters, $7=$ centroid of neutral hydrogen, $8=$ centroid of bright stars $(M<-5), 9=$ center of symmetry of $H_{I}$ rotation curve ('radio center' $C_{r}$ ), $10=$ center of symmetry of rotation curve of planetaries. 
(1955) simply to replace the actual asymmetric rotation curve by a fictitious, but more or less equivalent symmetric curve to which they could apply the standard analysis based on existing galaxy models which all assumed circular symmetry. The need for a dynamical theory of asymmetric barred systems was clearly recognized, however (de Vaucouleurs, 1960, p. 281).

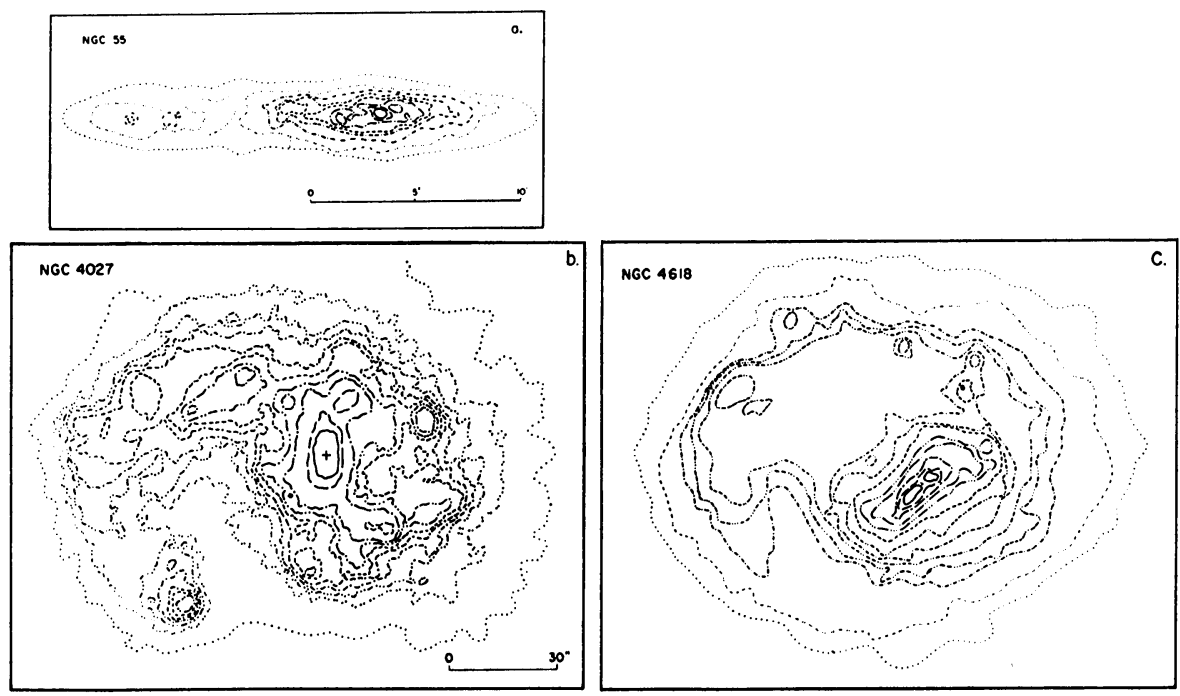

Fig. 3. Isophotes of three Magellanic barred spirals in blue light. - (a) NGC 55 (de Vaucouleurs, 1961 a); - (b) NGC 4027 (de Vaucouleurs et al., 1968); - (c) NGC 4618 (Bertola 1967). Note eccentric location of bar in flat disk component.

3. A plausible and still simple model, suggested by the surface photometry of systems such as the Large Cloud, NGC 55, 4027, 4618, etc. (Figures 3a, b, c) is shown in Figure 4; it consists of (1) a large oblate inhomogeneous spheroid of mass $M_{d}$ with axis ratio $c / a=0.2$ and some suitable density low following one of Perek's (1948, 1962) standard distributions, to approximate the main disk population of the Galaxy, and (2) a small prolate spheroid of axis ratio $c / a=5$ and mass $M_{\mathrm{b}} \simeq 0.1 M_{\mathrm{d}}$ approximating the bar.

This bar is displaced from the center of the large spheroid by a small distance $\Lambda$ (Figure 4), typically of the order of $1 \mathrm{kpc}$ in Magellanic spirals. How this situation arises in the first place is not our concern here since observation proves that it is present in nature. One might speculate that when primordial gas masses assemble to form a proto-galaxy there is no particular reason why the assemblage should have spherical or even circular symmetry; the amount of asymmetry may in fact determine the particular galaxy type formed.

4. Some of the properties of Magellanic barred spirals can be understood from the properties of test particle orbits in the gravitational field of the configuration described in Section 3. For example, Figure 5 shows the mean rotation curve for NGC 4027 


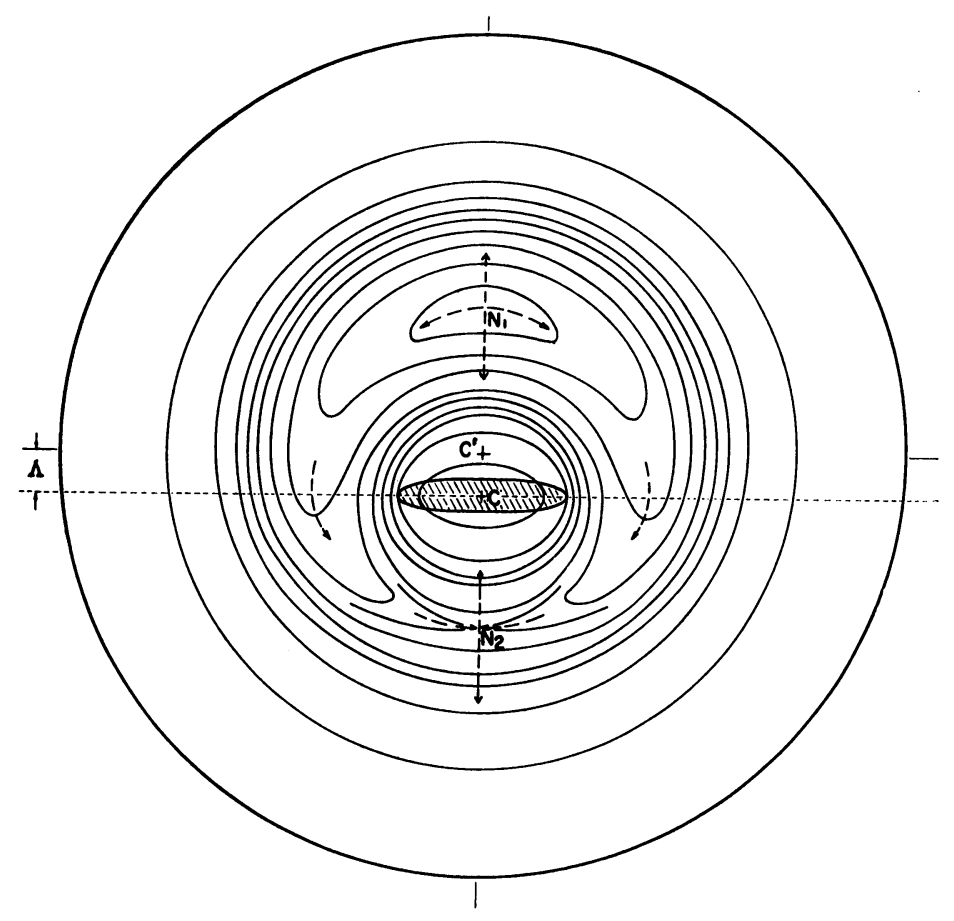

Fig. 4. Model of asymmetric barred spiral with equipotential lines. The inner prolate spheroid centered at $C$ (bar) is displaced by a distance $\Lambda$ from center $C^{\prime}$ of outer oblate spheroid (disk or main body). Neutral points are at $N_{1}$ and $N_{2}$. Compare Figure 3.

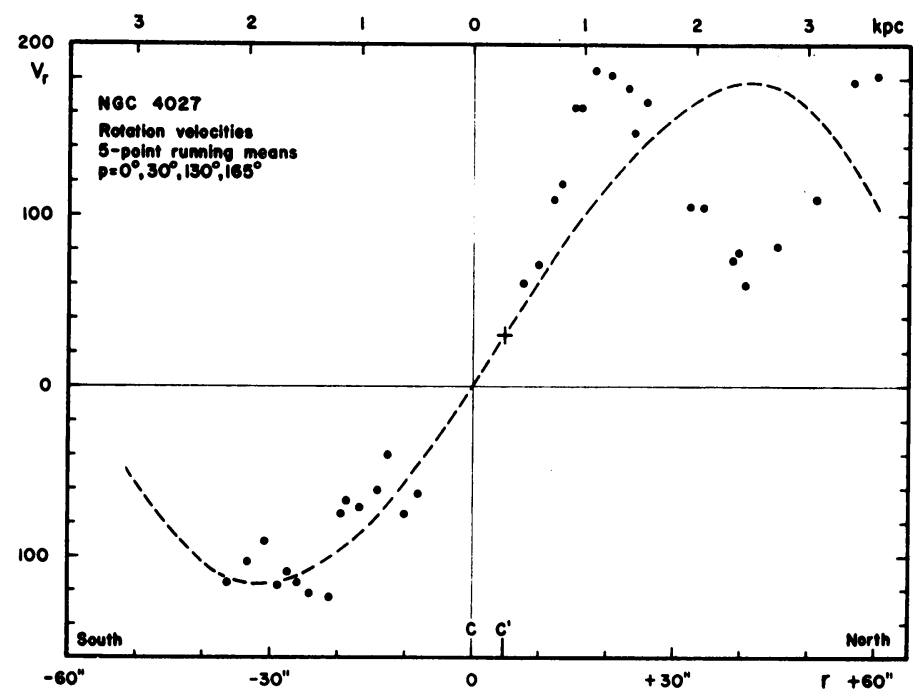

Fig. 5. The mean rotation curve of NGC 4027. The points are mean points from spectra in four position angles. The broken line is the best fit cubic to the mean points. $C$ is the bar center, and $C^{\prime}$ the center of symmetry of the cubic. 
(de Vaucouleurs et al., 1968); see also Figure 3b. On the North side of the bar (the major arm side), the mean rotational velocity appears to decrease from $180 \mathrm{~km} \mathrm{~s}^{-1}$ at $R=1.2 \mathrm{kpc}$ to $70 \mathrm{~km} \mathrm{~s}^{-1}$ at $R=2.4 \mathrm{kpc}$, and then increases again to $180 \mathrm{~km} \mathrm{~s}^{-1}$ at $R=3.6 \mathrm{kpc}(R=0$ is the bar center). There is no corresponding feature in the rotation curve on the other side of the bar.

To explain this feature, we use the model of Figure 4. It is assumed that the bar rotates (synchronously) about the disk center $C^{\prime}$ in such a way that the potential seen by an observer rotating with the bar is time-independent. We do not assume any particular functional dependence of the rotation period on $\Lambda$ and the masses of the bar and the disk, but prefer to estimate the significant dimensionless numbers from observation.

This is an unusual model for the potential field of a galaxy. It cannot be justified dynamically at this stage. We use it because it is simple and includes the relative displacement and orientation of the bar and disk indicated by optical and radio observations. This seems to us to be among the fundamental properties of Magellanic barred spirals.

The equipotentials of the gravitational + centrifugal force field for this model (as seen in a reference frame rotating with the bar) are shown in Figure 4: the dimensionless numbers for this model are appropriate to NGC 4027 (see de Vaucouleurs and Freeman, 1970). Note that (a) there are only two neutral points outside the bar: $N_{1}$ (stable) and $N_{2}$ (unstable); (b) the parameters for NGC 4027 locate $N_{1}$ at about $2 \mathrm{kpc}$ north of the bar center (cf. Figure 5).

There are two families $A$ and $B$ of periodic orbits around the stable neutral point $N_{1}$ (Figure 6). Calculation of the characteristic exponents for these orbits show that members of these families with amplitudes smaller than $A_{1}, B_{1}$ respectively (Figure 6)

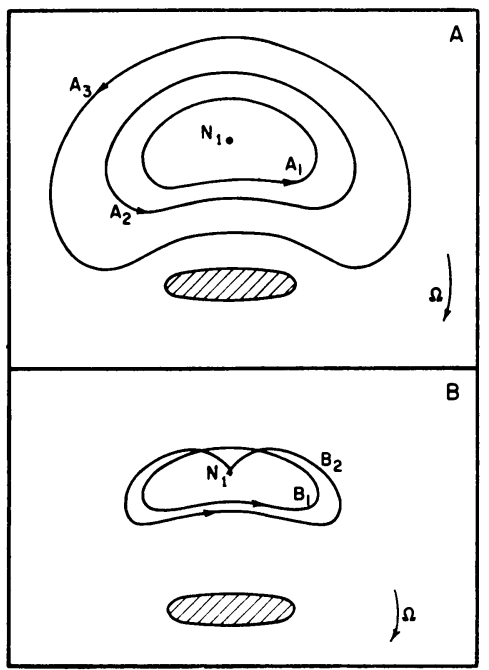

Fig. 6. Trajectories on the galactic plane for members of two families of periodic orbits near the neutral point $N_{1}$. The bar, and the sense of the rotation, are also shown. 
are stable. Surface of section analysis for values of the Jacobi integral corresponding to these stable orbits shows that they are stable to finite perturbations. This means that it is possible to trap a substantial amount of matter within the region enclosed by the orbit $A_{1}$; this matter circulates in a retrograde sense around $N_{1}$.

Now consider the rotation curve that would be observed for this model by an observer located at an infinite distance to the left-hand side of Figure 6; this location is appropriate to NGC 4027 (see de Vaucouleurs et al., 1968). For points in the region enclosed by $A_{1}$, the matter circulating around $N_{1}$ would cause an increase over the 'local circular velocity' in the apparent rotational velocity for $R<2 \mathrm{kpc}$, and a corresponding decrease for $R>2 \mathrm{kpc}$, with approximately the observed amplitude.

The neutral point $N_{2}$ is unstable. There are no stable periodic orbits corresponding to families $A$ and $B$ and there is no trapping of matter near $N_{2}$. The disturbance to the rotation curve then appears only on the $N_{1}$ side of the bar, as observed.

Full details of this work are given by de Vaucouleurs and Freeman (1970).

\section{Acknowledgements}

The research reported here has been supported in part by the U.S. National Science Foundation and by the Research Institute of the University of Texas.

\section{References}

Beale, J. S. and Davies, R. D.: 1969, Nature 221, 531.

Bertola, F.: 1967, Mem. Soc. Astron. Ital. 38, No. 2.

Crillon, R. and Monnet, G.: 1969, Astron. Astrophys. $2,1$.

Feast, M. W.: 1964, Monthly Notices Roy. Astron. Soc. 127, 195.

Gordon, K. J.: 1969, University of Michigan dissertation.

Kerr, F. J. and Vaucouleurs, G. de: 1955, Australian J. Phys. 8, 508.

Perek, L.: 1948, Contr. Astron. Inst. Masaryk 1, No. 6.

Perek, L. 1962, Adv. Astron. Astrophys. 1, 165.

Roberts, M. S.: 1968, Astrophys. J. 151, 117.

Robinson, B. J. and Van Damme, K. J.: 1966, Australian J. Phys. 19, 111.

Vaucouleurs, G. de: 1959a, Astrophys. J. 130, 728.

Vaucouleurs, G. de: 1959b, Handbuch der Physik 53, 275.

Vaucouleurs, G. de: 1960, Astrophys. J. 131, 265.

Vaucouleurs, G. de: 1961a, Astrophys. J. 133, 405.

Vaucouleurs, G. de: 1961b, Amer. Phil. Soc. Yearbook 1961, p. 268.

Vaucouleurs, G. and A. de: 1963, Astrophys. J. 137, 363.

Vaucouleurs, G. de and Freeman, K. C.: 1970, Vistas in Astronomy, in press.

Vaucouleurs, G. and A. de and Freeman, K. C.: 1968, Monthly Notices Roy. Astron. Soc. 139, 425. 